\title{
Esporotricose canina: relato de três casos
}

\author{
Canine sporotrichosis: report of three cases \\ Isabel Martins Madrid ${ }^{1}$, Ronaldo Santos Júnior ${ }^{2}$, Daiser Paulo Sampaio J r. ${ }^{3}$, Eduardo Negri Mueller ${ }^{4}$,

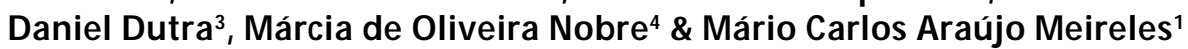

\begin{abstract}
RESUMO
A esporotricose é uma micose, subcutânea e zoonótica, causada pelo fungo dimórfico Sporothrix schenckii que afeta animais e humanos. A micose em cães era considerada rara devido aos poucos relatos descritos na literatura, no entanto, nos últimos anos observou-se um crescente registro de casos desta doença no Brasil. Neste estudo foram analisadas as características clínicas da esporotricose em três cães diagnosticados no Laboratório de Micologia (FV-UFPel), os quais apresentavam dispnéia, secreção nasal e espirros. Dois destes demonstravam também, lesões cutâneas ulceradas e crostosas no plano nasal. Embora um dos animais não apresentasse lesões cutâneas, os sinais respiratórios eram evidentes assim como a presença de linfadenopatia. Este estudo alerta para a ocorrência da esporotricose em cães na região de Pelotas e Rio Grande, e salienta para a similaridade das lesões observadas, com outras doenças infecciosas e neoplásicas, o que torna importante a realização de diagnóstico diferencial.
\end{abstract}

Descritores: cães, micose, Sporothrix schenckii, itraconazol.

\begin{abstract}
Sporotrichosis is a subcutaneous and zoonotic mycosis caused by dimorphic fungus Sporothrix schenckii that affects animals and humans. The disease in dogs was considered rare due to the few reports described in the literature, however, in the last years a crescent registration of cases of this mycosis was observed in Brazil. In this study the clinical characteristics of the sporotrichosis were analyzed in three dogs diagnosed at the Laboratório de Micologia (FV-UFPel), which presented dyspnea, nasal secretion and sneezes. Two of these also demonstrated ulcers and crusts in the nasal plan. Although one of the animals didn't present cutaneous lesions, the respiratory signs were evident as well as the lymphadenopathy presence. This alert study for the occurrence of the sporotrichosis in dogs in the region of Pelotas and Rio Grande, and accentuate for the similarity of the lesions observed, with other infectious and neoplastic diseases, what turns important the realization of diagnose differential.
\end{abstract}

Key words: dogs, mycosis, Sporothrix schenckii, itraconazole. 


\section{INTRODUÇÃO}

A esporotricose é uma micose de distribuição mundial, causada pelo fungo dimórfico Sporothrix schenckii que acomete uma grande variedade de espécies animais e o homem. O fungo pode ser encontrado no solo, associado com plantas e matéria orgânica em decomposição, sendo mais prevalente em áreas de clima tropical e subtropical [1].

A infecção ocorre, principalmente, pelo implante traumático do fungo na pele produzindo lesões nodulares e ulcerativas que drenam exsudato acastanhado levando a formação de crostas. A esporotricose pode apresentar-se nas formas cutânea fixa, linfocutânea e cutânea disseminada, raramente evoluindo para a forma sistêmica. Ocasionalmente pode ocorrer inalação de conídios de $S$. schenckii causando esporotricose pulmonar [4]. Esta micose tem sido frequentemente descrita na espécie felina, a qual é importante fonte de transmissão para humanos e outros animais $[7,11,13]$. Na espécie canina esta doença era considerada rara, no entanto, alguns autores têm alertado quanto à ocorrência desta micose no Rio de Janeiro, com mais de 44 casos descritos entre 1998 e 2006 [9,12]. Em vista do aumento de relatos de esporotricose em cães, este trabalho visa contribuir ao conhecimento da esporotricose no Brasil, descrevendo aspectos clínicos da esporotricose canina no sul do Rio Grande do Sul.

\section{RELATO DOS CASOS}

Durante o período de dois anos foram diagnosticados três casos de esporotricose canina (Figura 1) nas cidades de Pelotas (RS) e Rio Grande (RS), sendo colhidas amostras de tecido e swab de lesões, cavidade nasal, oral e ocular, para realização de exame micológico e histopatológico. Para a colheita destas amostras, os animais foram anestesiados e a biópsia cutânea foi realizada com auxílio de punch de $6 \mathrm{~mm}$.

As amostras encaminhadas ao Laboratório de Doenças Infecciosas (Setor de Micologia - FV-UFPel) foram processadas através do cultivo, em duplicata, em placas de Petri contendo ágar Sabouraud acrescido de cloranfenicol e cicloheximida, sendo incubadas a $25^{\circ} \mathrm{C}$ e $37^{\circ} \mathrm{C}$ por três semanas. Os fragmentos de tecido enviados ao Laboratório Regional de Diagnóstico (FV-UFPel) para exame histopatológico, foram fixados em formalina $10 \%$ e os cortes histológicos corados pelas técnicas de hematoxilina-eosina (HE) e Periodic Acid Schiff (PAS).
Os caninos eram machos com idade entre quatro e nove anos, um sem raça definida (C1), um de raça Pinscher (C2) e outro Pitbull (C3), os quais apresentavam sinais respiratórios caracterizados por espirros, secreção nasal sero-mucosa e dispnéia. A evolução dos sinais clínicos variou entre um mês e três anos, sendo que os animais, $\mathrm{C} 1$ e $\mathrm{C} 2$, apresentavam lesões cutâneas ulceradas, exsudativas e crostosas no plano nasal e comprometimento ósseo da região nasal. No C1, também foi observado lesões nodulares e ulceradas no membro anterior direito e no $\mathrm{C} 2$, linfadenite dos linfonodos submandibulares, além das lesões no plano nasal. Embora o C3 não apresentasse lesões cutâneas em nenhuma área corpórea, os sinais respiratórios eram evidentes assim como o aumento de volume dos linfonodos submandibulares com nódulos ao longo dos vasos linfáticos aferentes.

O diagnóstico de esporotricose foi estabelecido pelo isolamento do fungo Sporothrix schenckii nas amostras de tecido e/ou exsudato de todos os animais e na amostra de cavidade oral do $\mathrm{C} 1$, sendo o dimorfismo confirmado pela obtenção de colônias a $37^{\circ} \mathrm{C}$, cremosas e de coloração branco-amarelada, e a $25^{\circ} \mathrm{C}$, pregueadas, membranosas e de coloração inicialmente creme tornando-se acastanhadas após 15 dias de incubação. Posterior à confirmação do diagnóstico iniciou-se terapia antifúngica com itraconazol na dose de $10 \mathrm{mg} / \mathrm{kg}$, via oral, uma vez ao dia, por um período mínimo de três meses. Os animais demonstraram melhora do quadro clínico após 20 dias de tratamento, sendo observada a cura clínica dos animais ao final do período previamente determinado.

No exame histopatológico pela coloração de HE observou-se a presença de piogranulomas focais ou multifocais compostos por macrófagos, neutrófilos, células gigantes, epitelióides e necrose central, determinando uma dermatite piogranulomatosa profunda. Apenas na amostra do $\mathrm{C} 2$ foi visualizada a presença de estruturas ovais a alongadas, PAS positivas, compatíveis com S. schenckii.

\section{DISCUSSÃO}

A esporotricose canina ocorre principalmente nas formas cutânea fixa e linfocutânea com lesões localizadas no focinho e/ou membros [2,10]. No entanto, a ocorrência nasal com ausência de lesões cutâneas não foi encontrada na literatura consultada, sendo este o primeiro relato. Os nódulos e úlceras decor- 
rentes da infecção fúngica são semelhantes aos que ocorrem em outras doenças infecciosas e neoplásicas. Santos et al. (2006) salientaram a importância da realização do exame laboratorial com isolamento do agente etiológico para o diagnóstico diferencial entre esporotricose e leishmaniose em caninos.

$\mathrm{O}$ primeiro caso descrito de esporotricose canina foi em 1964, sendo que os animais apresentavam lesões circunscritas semelhantes àquelas causadas por dermatófitos [6]. Posteriormente, poucos casos foram relatados, mas nos últimos anos esta doença tem sido freqüentemente relacionada com a transmissão por felinos domésticos $[8,12,13]$. Neste estudo, verificou-se ainda, um caso de esporotricose canina transmitida por felino (I.M., resultados não publicados).
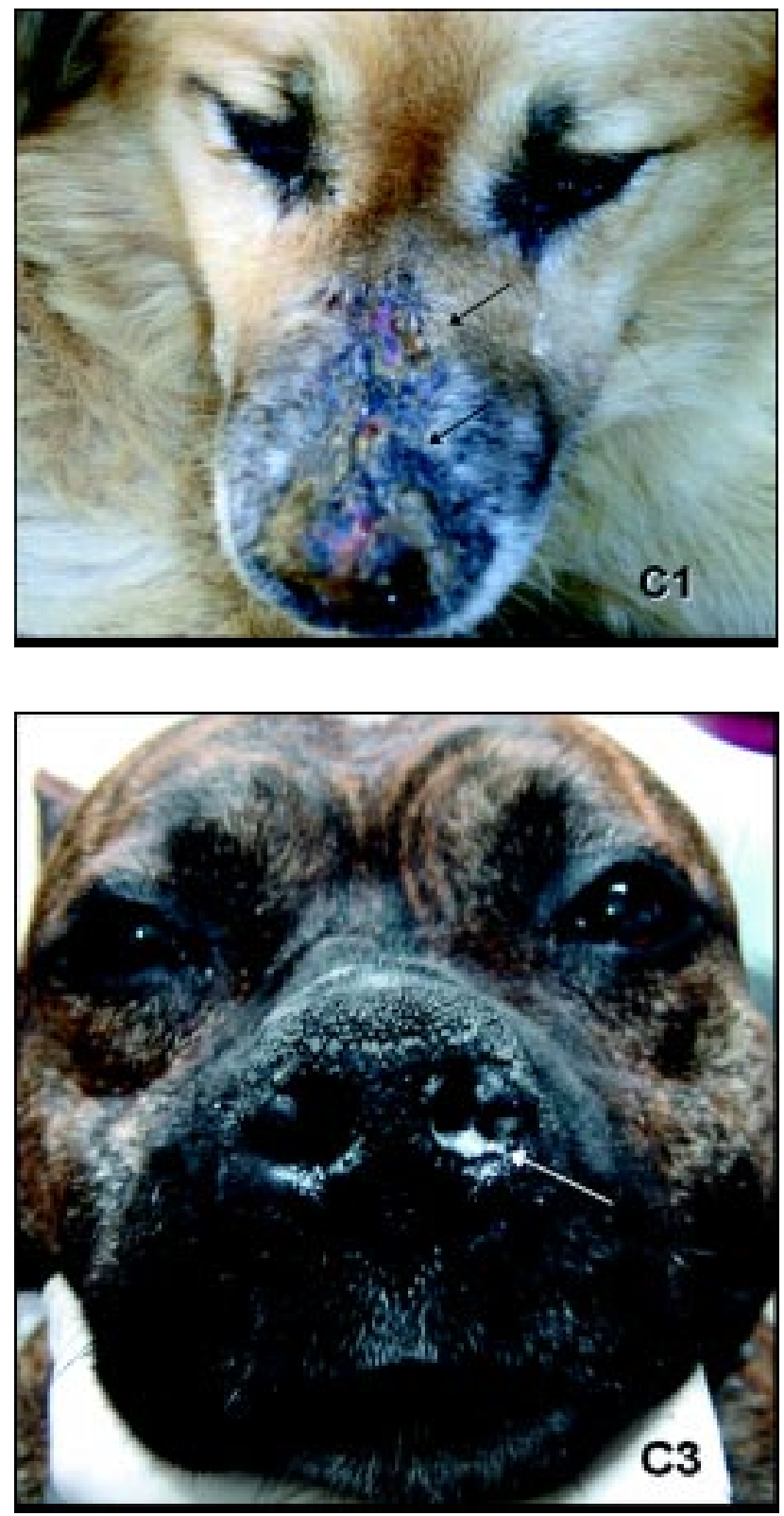

As colônias obtidas na análise micológica são semelhantes às descritas na literatura, no entanto, pode-se observar que as colônias provenientes de amostras de cães crescem mais lentamente e são menos pigmentadas que as colônias obtidas de amostras de felinos [1,4]. As alterações histopatológicas observadas neste estudo são comumente descritas em lesões de esporotricose, assim como a presença de poucas células leveduriformes ou ausência destas, em amostras provenientes de caninos [2,3,12].

Durante o tratamento não foram observados efeitos colaterais e os cães continuam sendo acompanhados para verificar a possível recidiva das lesões. Diversos fármacos são utilizados no tratamento da esporotricose, no entanto, o itraconazol tem demonstrado eficácia e segurança para o uso em diversas espécies [10,12].

Este estudo alerta para a ocorrência da esporotricose em cães na região sul do Rio Grande do Sul, e demonstra que as lesões de esporotricose em cães são variadas, podendo ser confundidas com outras enfermidades que possuem sinais clínicos semelhantes aos observados nestes relatos.

Agradecimentos. Ao Conselho Nacional de Desenvolvimento Científico e Tecnológico (CNPq - 151625/2004-2 e 474533/2004-2) e Coordenação de Aperfeiçoamento do Ensino Superior (CAPES) pelo suporte financeiro.

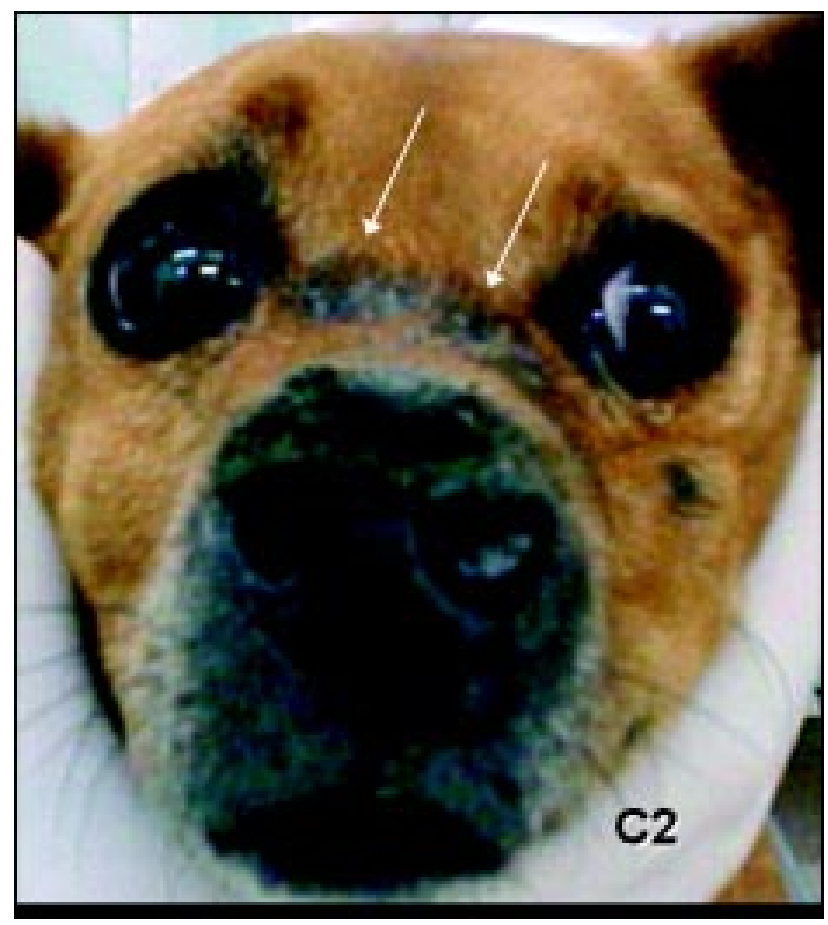

Figura 1. Presença de lesões crostosas no plano nasal (C1 e C2) e secreção sero-mucosa na cavidade nasal (C3) nos três cães com esporotricose. (Setor de Micologia, FV-UFPel, 2007). 


\section{REFERÊNCIAS}

1 Donadel K.W., Reinoso Y.D., Oliveira J.C. \& Azulay R.D. 1993. Esporotricose: revisão. Anais Brasileiro de Dermatologia . 68: $45-52$.

2 Farias M.R., Costa P.R.S., Franco S.R.V.S. \& Ferreira H. 1997. Esporotricose canina e felina. Cães \& Gatos. 66: $30-38$.

3 Freitas D.C., Moreno G., Saliba A.M.F., Cottino A.J. \& Mos E.M. 1965. Esporotricose em cães e gatos. Revista da Faculdade de Medicina Veterinária de São Paulo. 7:381-387.

4 Kwon-Chung K. \& Bennet J. 1992. Sporotrichosis. Medical Mycology, Philadelphia, p.707-729.

5 Larsson C.E., Dagli M.L.Z., Paula C.R. \& Michalany N.S. 1993. Esporotricose Canina - Relato de Caso Insólito.. In: $X V$ Congresso Brasileiro da ANCLIVEPA (Rio de Janeiro, Brasil)

6 Londero A.T., Castro R.M., Fischman O. 1964. Two cases of sporotrichosis in dog in Brazil. Sabouraudia. 3: $273-274$.

7 Nobre M.O., Castro A.P., Caetano D., Souza L.L., Meireles M.C.A. \& Ferreiro L. 2001. Recurrence of sporotrichosis in cats with zoonotic involvement. Revista Iberoamericana de Micologia. 18: 137-140.

8 Roeder L.D., Kasecker G.G., Brito H.F.V., Cit L.H.A., Tranquilim M.V., Ferrari M.V., Franco M.B. \& Sincero P.C. 2002. Esporotricose canina - relato de caso. In: Congresso Brasileiro de Especialidades em Medicina Veterinária (Curitiba, Brasil). p.175.

9 Santos I.B., Schubach T.M.P., Leme L.R.P., Okamoto T., Figueiredo F.B., Pereira S.A., Quintella L.P., Madeira M.F., Coelho F., Reis R.S. \& Schubach A.O. 2006. Sporotrichosis - The main differential diagnosis with tegumentary leishmaniosis in dogs from Rio de Janeiro, Brazil. Veterinary Parasitology. [in press]

10 Schubach T.M.P \& Schubach A.O. 2000. Esporotricose em gatos e cães - revisão. Clínica Veterinária. 5: 21-24.

11 Schubach T.M., Schubach A., Okamoto T., Barros M., Figueiredo F.B., Cuzzi T., Fialho-Monteiro P.C., Perez R.S. \& Wanke B. 2004. Evaluation of an epidemic of sporotrichosis in cats: 347 cases (1998-2001). Journal of the American Veterinary Medical Association. 224: 1623-1629.

12 Schubach T.M.P., Schubach A., Okamoto T., Barros M.B.L., Figueiredo F.F., Cuzzi-Maya T., Pereira S.A., Santos I.B., Paes R.A., Paes-Leme L.R. \& Wanke B. 2006. Canine sporotrichosis in Rio de Janeiro, Brazil: clinical presentation, laboratory diagnosis and therapeutic response in 44 cases (1998-2003). Medical Micology. 44: 87-92.

13 Xavier M.O., Nobre M.O., Sampaio Jr D.P.,Antunes T.A., Nascente P.S., Sória F.B.A. \& Meireles M.C.A. 2004. Esporotricose felina com envolvimento humano na cidade de Pelotas, RS, Brasil. Ciência Rural. 34: 1961-1963. 